\title{
Discharge Hemoglobin Association with Long-Term Outcomes of ST-Elevation Myocardial Infarction Patients Undergoing Primary Percutaneous Coronary Intervention
}

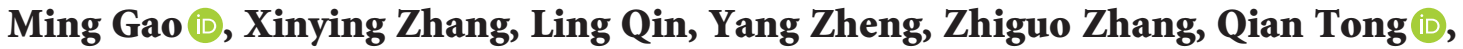 \\ and Hang Li \\ Cardiovascular Center, The First Hospital of Jilin University, Changchun 130021, China \\ Correspondence should be addressed to Qian Tong; tongqian187@aliyun.com and Hang Li; lihang62698@163.com
}

Received 12 September 2019; Revised 11 December 2019; Accepted 3 January 2020; Published 1 March 2020

Academic Editor: James C. Coons

Copyright (c) 2020 Ming Gao et al. This is an open access article distributed under the Creative Commons Attribution License, which permits unrestricted use, distribution, and reproduction in any medium, provided the original work is properly cited.

Background. Anemia following acute myocardial infarction (AMI) is associated with poor outcomes. While previous studies in patients with AMI have focused on anemia at admission, we hypothesized that hemoglobin $(\mathrm{Hb})$ decline during hospitalization and lower discharge $\mathrm{Hb}$ would be associated with greater long-term mortality in patients undergoing primary percutaneous coronary intervention (PCI) for ST-segment elevation myocardial infarction (STEMI). Methods. We analyzed records of 983 STEMI patients who were treated with primary PCI. The primary end point was all-cause mortality at 1 year and 2 years. The relationship between discharge $\mathrm{Hb}$ levels, decline in $\mathrm{Hb}$ levels, bleeding event classification, and all-cause mortality was determined. Results. Overall, $16.4 \%$ of patients had bleeding events, which were classified by the Thrombolysis in Myocardial Infarction (TIMI) score as $7 \%$ minimal, $8.6 \%$ minor, and $0.9 \%$ major. No significant gastrointestinal bleed and cerebral hemorrhage occurred in hospitals among these patients. The incidence rate of the 2-year all-cause mortality increased with severity of the bleeding event score $(8.78 \%$ for no bleeding vs. $11.59 \%$ for minimal bleeding vs. $20.24 \%$ for minor bleeding vs. $55.56 \%$ for major bleeding, $P<0.001)$. Discharge $\mathrm{Hb}$ was significantly associated with 2 -year mortality in an unadjusted model (hazard ratio $(\mathrm{HR})$ per $1 \mathrm{~g} / \mathrm{L}$ decrease in discharge $\mathrm{Hb}=1.020,95 \%$ confidence interval $(\mathrm{CI}): 1.006-1.034, P=0.004)$ and in a confounder-adjusted model (HR per $1 \mathrm{~g} / \mathrm{L}$ decrease in discharge $\mathrm{Hb}=1.024,95 \% \mathrm{CI}$ : 1.011-1.037, $P<0.001$ ). The odds ratio (OR) for all-cause mortality at 2 years for participants with $\mathrm{Hb}$ below the twentieth percentile was 3.529 (95\% CI: 1.976-6.302) and 2.968 (95\% CI: 1.614-5.456) after adjustment for age and gender and 2.485 (95\% CI: 1.310-4.715) after adjustment for all covariates. Conclusions. In this population of patients hospitalized for STEMI, all-cause mortality increased with lower discharge Hb, and discharge $\mathrm{Hb}$ was a significant predictor of mortality risk.

\section{Introduction}

Anemia following acute myocardial infarction (AMI) is associated with poorer outcomes relative to nonanemic AMI patients. Furthermore, anemia is more frequently encountered in patients hospitalized for cardiac events than in the general population $[1,2]$, ranging from $11 \%$ to $38 \%$ in patients with AMI $[3,4]$.

During myocardial infarction, oxygen delivery to the heart is reduced. In AMI, the amount of oxygen delivered to the heart is further decreased, and this insufficient oxygenation jeopardizes the myocardium. After recovery, cardiac output is increased to maintain adequate systemic oxygen delivery, a compensation that might impair recovery. Indeed, previous studies show that anemic patients experience worse outcomes after percutaneous coronary intervention (PCI; e.g., increased risks for stent thrombosis, longterm mortality, and bleeding) than myocardial infarction patients without anemia $[5,6]$.

The majority of previous studies in patients with AMI have focused on anemia present at admission [6,7], and little is known regarding hemoglobin $(\mathrm{Hb})$ levels at discharge among patients who undergo primary PCI for ST-segment elevation myocardial infarction (STEMI) or the change in 
$\mathrm{Hb}$ levels and mortality among patients' subsequent outcomes. Because of the additional cardiac strain caused by anemia after STEMI, we hypothesized that $\mathrm{Hb}$ decline and lower discharge $\mathrm{Hb}$ would be associated with a steeper functional decline in patients undergoing primary PCI for STEMI.

\section{Methods}

2.1. Study Population. We conducted a retrospective analysis of a cohort of consecutive patients with STEMI who underwent primary PCI in the Cardiology Department at the First Hospital of Jilin University between January 1, 2014, and December 31, 2015. The demographic, clinical, and angiographic characteristics of the patients had been prospectively collected in the department's electronic medical records, which store all patient-related health information obtained at primary care. Diagnosis of STEMI was consistent with the European Society of Cardiology/American College of Cardiology consensus document [8]. Patients who met at least 2 of the following criteria were included: characteristic severe chest pain or other symptoms suggestive of ischemia, electrocardiographic changes, and/or cardiac troponin value above the ninety-ninth percentile reference limits. A total of 1,615 STEMI patients who underwent emergency primary PCI were examined and, after excluding those who received emergency coronary artery bypass graft surgery, were diagnosed with thrombolysis or given only medical treatment or had unavailable $\mathrm{Hb}$ data at baseline and before discharge; 983 consecutive patients were included in our study. No significant gastrointestinal bleed and cerebral hemorrhage occurred in hospitals among these patients, and gastrointestinal bleed and cerebral hemorrhage were implicitly excluded from this study because patients with bleeding events are less likely to undergo PCI. The primary end point of the study was the occurrence of death. Discharge $\mathrm{Hb}$ was a covariate variable. In-hospital bleeding episodes were classified using the Thrombolysis in Myocardial Infarction (TIMI) criteria [9]. TIMI major bleeding was defined as intracranial hemorrhage or a decrease in $\mathrm{Hb}$ from admission to discharge greater than or equal to $5 \mathrm{~g} / \mathrm{dL}$. Minor bleeding was defined as a $3-5 \mathrm{~g} / \mathrm{dL}$ decline in $\mathrm{Hb}$, and minimal bleeding was defined as a less than $3 \mathrm{~g} / \mathrm{dL}$ decline in $\mathrm{Hb}$.

The study protocol was approved by the Ethics Review Board of the First Hospital of Jilin University (No. 2016263), and all participating patients approved the research protocols.

2.2. Laboratory Data. Peripheral blood samples were obtained at the time of hospital admission prior to performing primary PCI. The blood samples were used for routine blood chemistry and blood counts such as hemoglobin, total cholesterol (TC), creatinine, and triglyceride (TG) at the certified laboratory department of the First Hospital of Jilin University. The primary predictor variable for all analyses was $\mathrm{Hb}$ data following admission and before discharge. Discharge $\mathrm{Hb}$ was defined as the last $\mathrm{Hb}$ value $(\mathrm{g} / \mathrm{dL})$ obtained within 48 hours of discharge from the hospital.
2.3. Protocol and Definition. All patients were pretreated with a loading dose of $300 \mathrm{mg}$ aspirin, $600 \mathrm{mg}$ clopidogrel, and $1000 \mathrm{IU} / \mathrm{L}$ intravenous unfractionated heparin. Coronary angiography was performed using standard techniques. Thrombus aspiration, stent type, predilation, postdilation, and placement of a temporary pacemaker or intra-aortic balloon pump were decided at the surgeon's discretion. Following PCI, dual antiplatelet therapy (DAPT) with aspirin $(100 \mathrm{mg} /$ day) and clopidogrel (75 mg/day) was recommended in all patients with successful implantation of drug-eluting stents. Current American College of Cardiology/American Heart Association (ACC/AHA) [10] and European Society of Cardiology (ESC) guidelines [11] recommend DAPT of aspirin plus a P2Y12 inhibitor for at least 12 months or longer after implantation of drug-eluting stents in patients with acute coronary syndrome. During the time frame in which our study was conducted, our center recommended 18 months of DAPT.

2.4. Follow-Up. The patients' clinical courses were monitored by telephone interviews every six months after discharge and a comprehensive review of electronic medical records from our outpatient clinic. All patients were monitored over a period of 2 years or until death whichever occurred first. In the case of patients who were lost to followup, we contacted the patients' families or used the Social Security Administration Death Master File to confirm mortality.

2.5. Statistical Analysis. Characteristics of groups with or without $\mathrm{Hb}$ decline at baseline were compared by chisquared tests, independent-group $t$-tests, and Wilcoxon rank-sum tests, depending on the variable levels and distribution of measurements after testing for normality by the Kolmogorov-Smirnov test. The missing rates were less than $5 \%$ for each variable, and the attribute values were missing at random. The default missing-data analysis was completecase analysis. Cox multiple regression analysis was performed to evaluate the independent contributions of baseline clinical characteristics, medical history, and laboratory data to the occurrence of all-cause mortality at 2 years with forward stepwise selection. Patients were grouped according to the quintile of discharge $\mathrm{Hb}$ to permit comparison of the relationship with 2-year outcomes between the groups (quintile 1: 5.4-11.3 g/dL; quintile 2: 11.3-12.5 g/dL; quintile 3: $12.5-13.5 \mathrm{~g} / \mathrm{dL}$; quintile $4: 13.5-14.6 \mathrm{~g} / \mathrm{dL}$; quintile 5 : $14.6-18.8 \mathrm{~g} / \mathrm{dL})$. Hb levels of quintile 3 were used as a reference for odds ratio (OR) analyses. Logistic regression was performed to find whether a dose-response effect exists. Receiver-operating characteristic area under the curve (ROC-AUC) was constructed to further illuminate the best cutoff values per $1 \mathrm{~g} / \mathrm{L}$ decrease in discharge $\mathrm{Hb}$ to predict 2year all-cause mortality. Kaplan-Meier survival curve analysis compared survival among the severity of bleeding groups using the log-rank test. All analyses were conducted using Stata software, version 12 (Stata Corp., College Station, TX), and a two-sided $P<0.05$ was considered statistically significant. 


\section{Results}

3.1. Baseline Characteristics. The baseline characteristics of the 983 patients in the study population included a mean age of $61 \pm 12$ years; $66.9 \%$ of the participants were men (Figure 1 ) (Table 1). The majority of patients (93.2\%) underwent balloon angioplasty, and $86.9 \%$ received drug-eluting stent implantation. The mean admission hemoglobin was $14.1 \pm 2.1 \mathrm{~g} / \mathrm{dL}$, and the mean discharge hemoglobin was $12.9 \pm 2.0 \mathrm{~g} / \mathrm{dL}$. Overall, $16.4 \%$ of patients had bleeding events, classified under TIMI scores as $7.0 \%$ minimal, $8.6 \%$ minor, and $0.9 \%$ major. Among the total of nine patients $(0.9 \%)$ who had TIMI major bleeding events, four patients died before discharge and the remaining five experienced gradual reductions in $\mathrm{Hb}$ (the lowest of which was $>9.3 \mathrm{~g} / \mathrm{dL}$ ) without an additional significant clinical bleed in hospitals. Nonetheless, this group experienced an overall decline of health due to multiple concurrent diseases such as congestive heart failure, chronic renal failure, or pneumonia. Of the patients who died, prior to discharge, one case was complicated by acute renal insufficiency and one by the combination of pneumonia, acute respiratory failure, and ventricular arrhythmia. For both of these patients, $\mathrm{Hb}$ fluctuated between 7.4 and $8.7 \mathrm{~g} / \mathrm{dL}$ after surgery but was lowest $(5.4 \mathrm{~g} / \mathrm{dL})$ on the day of death. The other two patients who died after surgery had $\mathrm{Hb}$ values greater than 9.0 throughout the postsurgical period. None of these four patients received transfusion therapy.

Because pharmacological therapy could indeed have affected discharge $\mathrm{Hb}$ values, we controlled for pharmacological factors in the statistical analysis (Table 1). Aspirin, clopidogrel, and heparin therapy was initiated in all STEMI patients. The glycoprotein IIb/IIIa inhibitor tirofiban hydrochloride was administrated in patients with serious, angiography-proven thrombus burden. There were no differences between two groups in in-hospital pharmacological therapy.

3.2. Association between Bleeding and Mortality. During the 2-year follow-up period, all-cause mortality was $8.96 \%$ (89 deaths) at 1 year and $10.39 \%$ (103 deaths) at 2 years. There was a negative relation between survival and anemia severity. The 2-year all-cause mortality was significantly higher along with lower discharge $\mathrm{Hb}$ quintiles $(P<0.001$, Figure 2$)$. In Kaplan-Meier survival curve analysis, the incidence rate of 2-year all-cause mortality was significantly higher for TIMI minor and major bleeding events than for patients with no bleeding events $(8.78 \%$ for no bleeding vs. $11.59 \%$ for TIMI minimal bleeding vs. $20.24 \%$ for TIMI minor bleeding vs. $55.56 \%$ for TIMI major bleeding, $P<0.001$, Figure 3 ).

3.3. Association between Discharge $\mathrm{Hb}$ and Mortality. According to Cox proportional-hazards regression models, discharge $\mathrm{Hb}$ was significantly associated with 2-year mortality in the unadjusted model (hazard ratio (HR) per $1 \mathrm{~g} / \mathrm{dL}$ decrease in discharge $\mathrm{Hb}=1.020,95 \% \mathrm{CI}$ : $1.006-1.034, P=0.004)$ and remained significant after adjustment for potential confounders (HR per $1 \mathrm{~g} / \mathrm{dL}$ decrease in discharge $\mathrm{Hb}=1.024,95 \% \mathrm{CI}: 1.011-1.037, P<0.001)$. In multivariable models adjusting for patients' demographic and clinical characteristics, discharge $\mathrm{Hb}$, older age, female sex, prior myocardial infarction, chronic kidney disease, Killip classification $>2$, and absence of drug-eluting stent implantation were independent predictors for 2-year mortality (Figure 4).

3.4. Hb Stratification and Clinical Outcomes. The OR for allcause mortality at 2 years for participants with discharge $\mathrm{Hb}$ below the twentieth percentile was 3.529 (95\% CI: 1.976-6.302) and 2.968 (95\% CI: 1.614-5.456) after adjustment for age and sex and 2.485 (95\% CI: 1.310-4.715) after adjustment for all covariables (Table 2). Using a $2.6 \mathrm{~g} /$ $\mathrm{dL}$ decline in $\mathrm{Hb}$ as a cutoff for ROC-AUC, discharge $\mathrm{Hb}$ predicted 2-year mortality with a sensitivity of $86.55 \%$ and specificity of $80.16 \%$ (ROC-AUC: $0.550, \quad 95 \% \quad \mathrm{CI}$ : $0.484-0.625, P=0.034)$.

\section{Discussion}

In this population of patients hospitalized for STEMI, allcause mortality increased with severity of bleeding events, and discharge $\mathrm{Hb}$ was a statistically significant predictor of mortality risk.

Published studies focus on the relationship between anemia and outcomes in patients presenting with acute coronary syndromes and/or undergoing revascularization, demonstrating that anemia measured at a single time point is associated with worse outcomes [12-14]. In contrast, few studies have evaluated patients with STEMI. Therefore, the aim of the current analysis was to assess the relationship between discharge $\mathrm{Hb}$ and decline in $\mathrm{Hb}$ between admission and discharge with long-term, all-cause mortality in patients undergoing PCI for STEMI.

Anemia significantly decreases oxygen delivery to the myocardium downstream of coronary stenosis and increases myocardial oxygen demand by necessitating a higher stroke volume and heart rate to maintain adequate systemic oxygen delivery [15-17]. In STEMI, even mildly reduced $\mathrm{Hb}$ concentrations at the abrupt onset of coronary occlusion may significantly attenuate the ability of collateral flow from nearby patent vessels to limit the extent of myocardial necrosis and peri-infarct ischemia. The combination of these processes may explain the pathophysiology underlying the progressively poorer outcomes we observed in more severe bleeding events and lower discharge $\mathrm{Hb}$ concentrations. Furthermore, discharge $\mathrm{Hb}$ is an elegant marker for prognosis because it is routinely measured in patients hospitalized with AMI, and it is potentially modifiable with treatment. Notably, many patients in our cohort (16.4\%) had some decline in their $\mathrm{Hb}$ during AMI admission which is a high figure than expected.

Furthermore, we subdivided patients according to discharge $\mathrm{Hb}$ and found increased mortality risks in patients with only mildly reduced $\mathrm{Hb}$ concentration and a doseresponse effect that resulted in progressively lower survival rates with more profound degrees of anemia. These effects may have been masked in previous studies, which generally only distinguish between patients with and without anemia. 


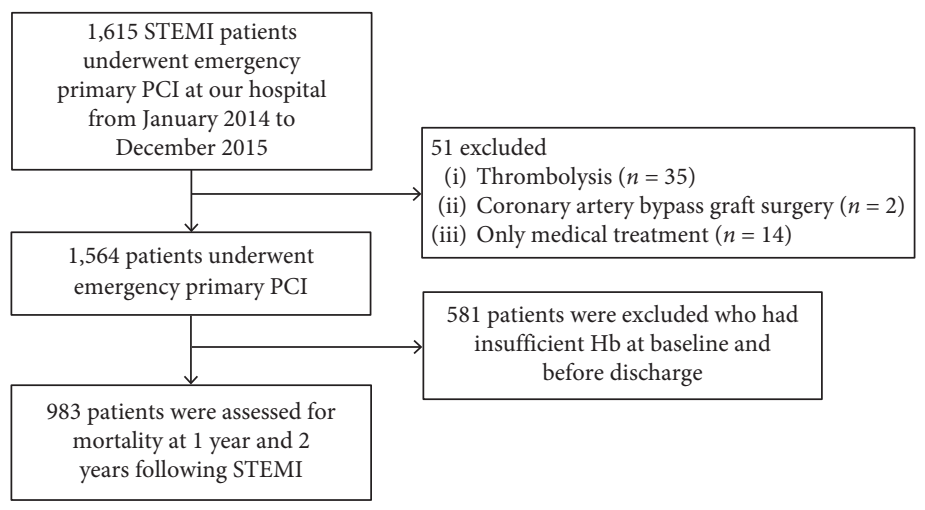

Figure 1: Flow chart of selection.

TABLE 1: Baseline characteristics of participants (overall and by presence/absence of bleeding events).

\begin{tabular}{|c|c|c|c|c|}
\hline Characteristics & $\begin{array}{l}\text { Overall }(n=983), n(\%) \\
\quad \text { or mean } \pm \text { SD }\end{array}$ & $\begin{array}{c}\text { Without Hb decline } \\
(n=821), n(\%) \text { or mean } \pm \mathrm{SD}\end{array}$ & $\begin{array}{l}\text { Hb decline }(n=162), n \\
(\%) \text { or mean } \pm \text { SD }\end{array}$ & $\begin{array}{c}P \text { value (without } \mathrm{Hb} \\
\text { decline vs. } \mathrm{Hb} \text { decline) }\end{array}$ \\
\hline Age (years) & $61 \pm 12$ & $61 \pm 12$ & $62 \pm 11$ & 0.470 \\
\hline Male gender & $658(66.9)$ & $551(67.1)$ & $107(66.0)$ & 0.793 \\
\hline \multicolumn{5}{|l|}{ Medical history } \\
\hline Diabetes mellitus & $251(25.5)$ & $209(25.5)$ & $42(25.9)$ & 0.900 \\
\hline Hypertension & $462(47.0)$ & $384(46.8)$ & $78(48.2)$ & 0.748 \\
\hline Previous PCI & $33(3.4)$ & $27(3.3)$ & $6(3.7)$ & 0.789 \\
\hline Atrial fibrillation & $65(6.6)$ & $50(6.1)$ & $15(9.3)$ & 0.138 \\
\hline $\begin{array}{l}\text { Peripheral vascular } \\
\text { disease }\end{array}$ & $9(0.9)$ & $4(0.5)$ & $5(3.1)$ & 0.008 \\
\hline $\begin{array}{l}\text { Prior myocardial } \\
\text { infarction }\end{array}$ & $34(3.5)$ & $28(3.4)$ & $6(3.7)$ & 0.852 \\
\hline $\begin{array}{l}\text { Prior cerebrovascular } \\
\text { event }\end{array}$ & $55(5.6)$ & $40(4.9)$ & $15(9.3)$ & 0.026 \\
\hline Chronic kidney disease & $45(4.6)$ & $36(4.4)$ & $9(5.6)$ & 0.515 \\
\hline Arrhythmia (VT/VF) & $82(8.3)$ & $65(7.9)$ & $17(10.5)$ & 0.278 \\
\hline $\begin{array}{l}\text { Infarct location by } \\
\text { electrocardiogram }\end{array}$ & & & & 0.269 \\
\hline Inferior & $285(29.0)$ & $230(28.0)$ & $55(34.0)$ & \\
\hline Anterior & $466(47.4)$ & $392(47.8)$ & $74(45.7)$ & \\
\hline Lateral & $232(23.6)$ & $199(24.2)$ & $33(20.4)$ & \\
\hline Killip classification & & & & 0.018 \\
\hline $\mathrm{I}$ & $613(62.4)$ & $522(63.6)$ & $91(56.2)$ & \\
\hline II & $205(20.9)$ & $175(21.3)$ & $30(18.5)$ & \\
\hline III & $52(5.3)$ & $39(4.8)$ & $13(8.0)$ & \\
\hline IV & $113(11.5)$ & $85(10.4)$ & $28(17.3)$ & \\
\hline \multicolumn{5}{|l|}{ Laboratory data } \\
\hline $\begin{array}{l}\text { Total cholesterol (mmol/ } \\
\mathrm{L})\end{array}$ & $4.7 \pm 1.2$ & $4.7 \pm 1.2$ & $4.8 \pm 1.4$ & 0.123 \\
\hline Triglycerides $(\mathrm{mmol} / \mathrm{L})$ & $1.7 \pm 1.1$ & $1.7 \pm 1.1$ & $1.9 \pm 1.3$ & 0.211 \\
\hline $\begin{array}{l}\text { Admission hemoglobin } \\
(\mathrm{g} / \mathrm{dl})\end{array}$ & $14.1 \pm 2.1$ & $13.9 \pm 2.1$ & $15.0 \pm 2.1$ & $<0.001$ \\
\hline $\begin{array}{l}\text { Discharge hemoglobin } \\
(\mathrm{g} / \mathrm{dl})\end{array}$ & $12.9 \pm 2.0$ & $13.1 \pm 1.9$ & $11.7 \pm 2.1$ & $<0.001$ \\
\hline Method of reperfusion & & & & 0.897 \\
\hline Balloon angioplasty & $916(93.2)$ & $767(93.4)$ & $149(92.0)$ & \\
\hline $\begin{array}{l}\text { Drug-eluting stent } \\
\text { implantation }\end{array}$ & $854(86.9)$ & $714(87.0)$ & $140(86.4)$ & \\
\hline Thrombus aspiration & $120(12.2)$ & $91(11.1)$ & $29(17.9)$ & 0.015 \\
\hline Temporary pacemaker & $67(6.8)$ & $52(6.3)$ & $15(9.3)$ & 0.177 \\
\hline Number of stents & $1.1 \pm 0.7$ & $1.1 \pm 0.7$ & $1.1 \pm 0.7$ & 0.626 \\
\hline $\begin{array}{l}\text { Intra-aortic balloon } \\
\text { pump }\end{array}$ & $8(0.8)$ & $7(0.9)$ & $1(0.6)$ & 0.610 \\
\hline Aspirin & $973(99.0)$ & $813(99.0)$ & $160(98.8)$ & 0.673 \\
\hline Clopidogrel & $977(99.4)$ & $815(99.3)$ & $162(100)$ & 0.597 \\
\hline Tirofiban hydrochloride & $112(11.4)$ & $95(11.6)$ & $17(10.5)$ & 0.787 \\
\hline
\end{tabular}

SD, standard deviation; PCI, percutaneous coronary intervention; VT/VF, ventricular tachycardia/ventricular fibrillation. 


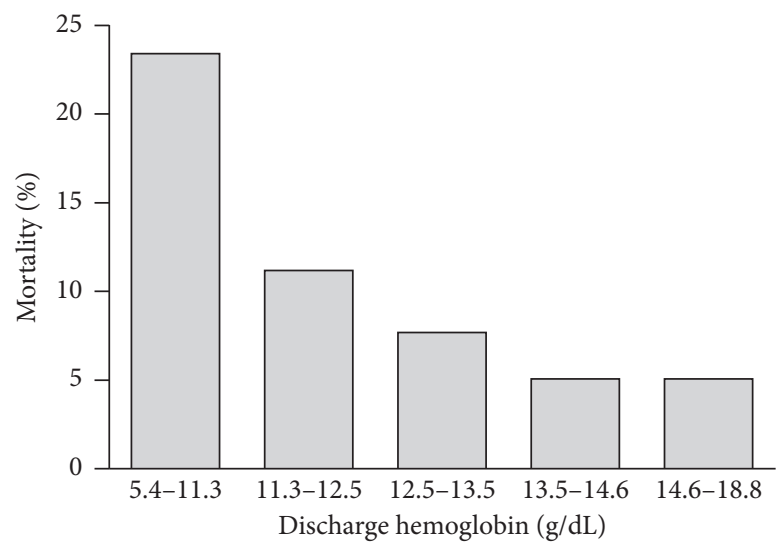

FIGURE 2: The incidence rate of unadjusted 2-year all-cause mortality was significantly higher for patients with lower discharge Hb.

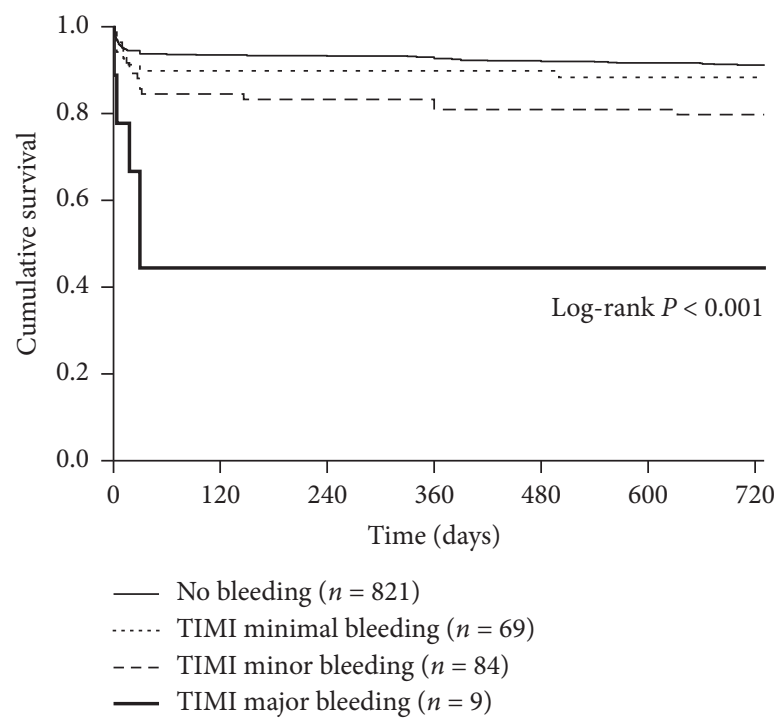

FIGURE 3: Kaplan-Meier survival curve analysis comparing cumulative incidence of all-cause mortality according to severity of bleeding events by Thrombolysis in Myocardial Infarction (TIMI) classification.

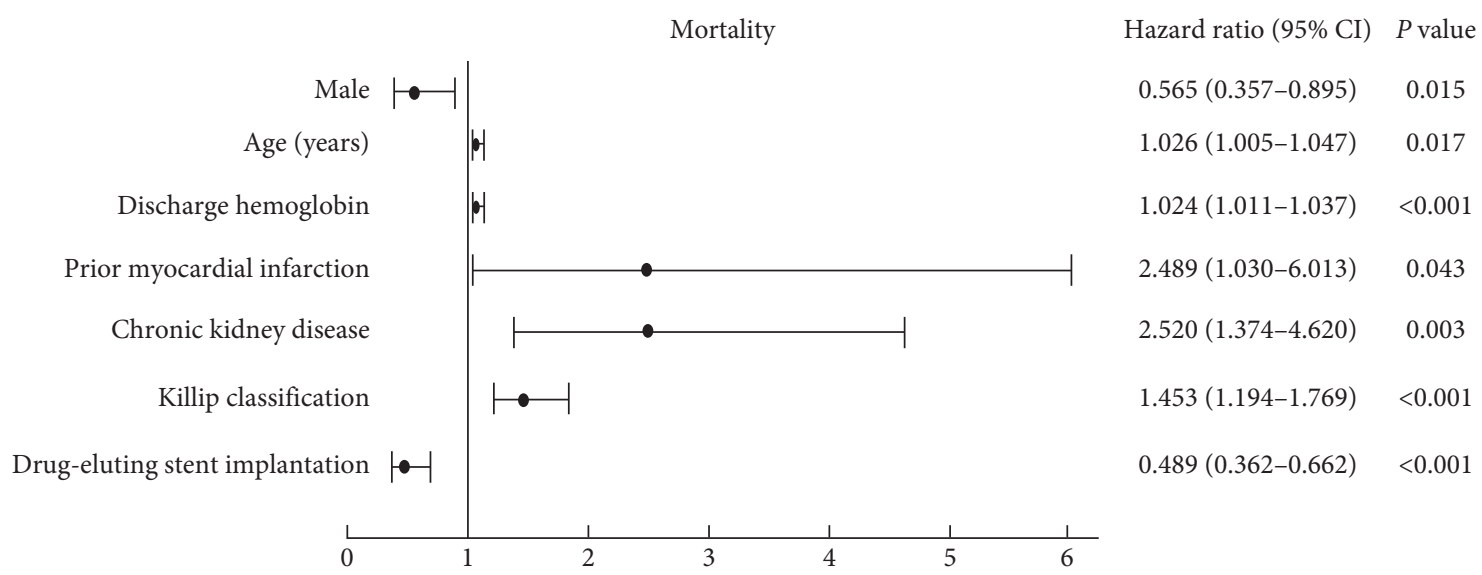

Figure 4: Multivariate predictors of 2-year mortality. CI, confidence interval.

In a meta-analysis of 233,144 patients with acute coronary syndromes, anemia was associated with a significantly increased risk of death or reinfarction [14]. In a study of 936 women undergoing evaluation for chest pain, $\mathrm{Hb}$ was an independent predictor of adverse cardiovascular outcomes, with a $20 \%$ increased risk for each $1 \mathrm{~g} / \mathrm{dL}$ decrement in $\mathrm{Hb}$ 
TABLe 2: Association of discharge hemoglobin with 2-year all-cause mortality.

\begin{tabular}{|c|c|c|c|c|c|c|c|c|c|}
\hline \multirow{2}{*}{ Hb concentration $(\mathrm{g} / \mathrm{dL})$} & \multicolumn{3}{|c|}{ Crude univariate model } & \multicolumn{3}{|c|}{ Adjusted for age } & \multicolumn{3}{|c|}{ Adjusted for all covariables } \\
\hline & HR & $95 \% \mathrm{CI}$ & $P$ value & OR & $95 \% \mathrm{CI}$ & $P$ value & OR & $95 \% \mathrm{CI}$ & $P$ value \\
\hline Qui & 3.529 & $76-6.302$ & $<0.001$ & 2.968 & $514-5.456$ & $<0.001$ & 2.485 & $.310-4.715$ & 0.005 \\
\hline Quintile 2: 11.3-12.5 & 1.418 & $0.731-2.750$ & 0 & 1.347 & $0.687-2.641$ & 0. & 1.220 & $0.604-2.465$ & 0.580 \\
\hline Quintile 3: $12.5-13.5^{\mathrm{a}}$ & 1 (ref) & & $<0$ & 1 (ref) & & $<0$. & 1 (ref) & & $<0.001$ \\
\hline Quintile 4: 13.5-14.6 & 0.716 & $0.329-1.560$ & 0.401 & 0.808 & 0.3 & 0.593 & 0.976 & $0.442-2.154$ & 0.952 \\
\hline Quintile 5: 14.6-18.8 & 0.659 & $0.296-1.466$ & 0.306 & 0.964 & $0.425-2.184$ & 0.930 & 1.200 & $0.522-2.757$ & 0.667 \\
\hline
\end{tabular}

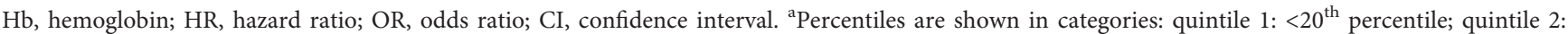
$20^{\text {th }}-<40^{\text {th }}$ percentile; quintile 3: $40^{\text {th }}-<60^{\text {th }}$ percentile; quintile $4: 60^{\text {th }}-<80^{\text {th }}$ percentile; quintile $5: \geq 80^{\text {th }}$ percentile.

[18]. Furthermore, in the elderly, per $1.0 \mathrm{~g} / \mathrm{dL}$ decrease in $\mathrm{Hb}$ over 3 years was associated with a 1.11-fold increased mortality over subsequent follow-up [19]. Although we demonstrated that the impact of $\mathrm{Hb}$ decline on mortality was greatest at lower discharge $\mathrm{Hb}$ concentrations, any decline greater than $2.6 \mathrm{~g} / \mathrm{dL}$ between admission and discharge conferred an increased risk of 2-year mortality.

Thus, prior research and our study demonstrate that both the absolute level of $\mathrm{Hb}$ and the change in $\mathrm{Hb}$ during hospitalization are important when evaluating the association of $\mathrm{Hb}$ concentrations with mortality. Prior treatment strategies to increase $\mathrm{Hb}$ concentration in patients with AMI and anemia might not significantly benefit longterm survival because guidelines do not specify $\mathrm{Hb}$ targets. Current clinical practice guidelines regarding blood transfusion are almost always indicated if $\mathrm{Hb}<6 \mathrm{~g} / \mathrm{dL}$ and rarely indicated if $\mathrm{Hb}>10 \mathrm{~g} / \mathrm{dL}$ [20]. For patients with $\mathrm{Hb}$ 6-10 g/dL, transfusion decisions depend on the extent of the blood loss, underlying cardiovascular disease, and overall clinical status. The state of anemia may represent an overall decline of health due to underlying comorbidities, and a simple preoperative correction of hemoglobin via transfusion may not positively affect the patient's operative course. However, other strategies can reduce perioperative risk in anemic patients, including the use of certain antiplatelet agents with care to avoid bleeding, using proton pump inhibitors to minimize upper gastrointestinal bleeding, and avoiding the use of glycoprotein IIb/ IIIa inhibitors. Given these findings, a prospective randomized clinical trial may be warranted to determine whether precise targeting of $\mathrm{Hb}$ levels improves outcomes in patients with AMI. Indeed, further study is necessary to assess whether intervening to increase $\mathrm{Hb}$ (outside of treating nutritional deficiencies), addressing blood loss, and managing chronic disease have any role in ameliorating adverse mortality outcomes after STEMI.

4.1. Study Limitations. There are several limitations to our study. Discharge hemoglobin was not routinely captured in our center. We excluded $36 \%$ of patients who did not have complete evaluation of discharge hemoglobin. The results may be biased due to the potential impact of data that were not available in our database, although there was continuous admission. Even though important comorbidities were included in our analysis, data on other measures of the overall health status (e.g., body mass index) were not available; thus, anemia may represent an overall decline of health due to underlying comorbidities. Despite the absence of this information, all categories of bleeding events and low discharge $\mathrm{Hb}$ predicted an increased risk for long-term mortality independent of a number of confounders in our study population. Prasugrel or ticagrelor was not available during the study period in our center, which prevents us from analyzing the impact of different P2Y12 inhibitors on $\mathrm{Hb}$ levels. We did not test routinely for drug use during follow-up; thus, the extent of adherence to the prescribed medical therapies could affect clinical outcomes, potentially causing us to overestimate mortality risk in patients with anemia. However, although anemia affected mortality, we cannot solely attribute the significantly worse long-term outcomes in patients with AMI to it.

\section{Conclusions}

In this population of patients hospitalized for STEMI, allcause mortality increased when discharge $\mathrm{Hb}$ was low and when bleeding events during hospitalization were more severe; lower discharge $\mathrm{Hb}$ was associated with long-term, all-cause mortality in patients undergoing PCI for STEMI.

\section{Data Availability}

The datasets used and analyzed during the current study are available from the corresponding author on request.

\section{Conflicts of Interest}

The authors declare that they have no conflicts of interest.

\section{Authors' Contributions}

Ming Gao and Xinying Zhang contributed equally to this article.

\section{Acknowledgments}

This work was supported by grant from the State's Key Project of Research and Development Plan (no. 2016YFC0900903), the Outstanding Young Talent Foundation of Jilin Province (no. 20190103085JH), and the Natural Science Foundation of Jilin Province (no. 20180101106JC). 


\section{References}

[1] A. C. Salisbury, K. P. Alexander, K. J. Reid et al., "Incidence, correlates, and outcomes of acute, hospital-acquired anemia in patients with acute myocardial infarction," Circulation: Cardiovascular Quality and Outcomes, vol. 3, no. 4, pp. 337-346, 2010.

[2] J. Anderson, L. G. Glynn, J. Newell, A. A. Iglesias, D. Reddan, and A. W. Murphy, "The impact of renal insufficiency and anaemia on survival in patients with cardiovascular disease: a cohort study," BMC Cardiovascular Disorders, vol. 9, no. 1, p. 51, 2009.

[3] J. O. Younge, S. T. Nauta, K. M. Akkerhuis, J. W. Deckers, and R. T. van Domburg, "Effect of anemia on short- and long-term outcome in patients hospitalized for acute coronary syndromes," The American Journal of Cardiology, vol. 109, no. 4, pp. 506-510, 2012.

[4] M. G. Colombo, I. Kirchberger, U. Amann et al., "Association between admission anemia and long-term mortality in patients with acute myocardial infarction: results from the MONICA/KORA myocardial infarction registry," $B M C$ Cardiovascular Disorders, vol. 18, no. 1, p. 50, 2018.

[5] B. J. Doyle, C. S. Rihal, D. A. Gastineau, and D. R. Holmes Jr., "Bleeding, blood transfusion, and increased mortality after percutaneous coronary intervention," Journal of the American College of Cardiology, vol. 53, no. 22, pp. 2019-2027, 2009.

[6] V. Kunadian, R. Mehran, A. M. Lincoff et al., "Effect of anemia on frequency of short- and long-term clinical events in acute coronary syndromes (from the acute catheterization and urgent intervention triage strategy trial)," The American Journal of Cardiology, vol. 114, no. 12, pp. 1823-1829, 2014.

[7] T. Kurek, R. Lenarczyk, J. Kowalczyk et al., "Effect of anemia in high-risk groups of patients with acute myocardial infarction treated with percutaneous coronary intervention," The American Journal of Cardiology, vol. 105, no. 5, pp. 611-618, 2010.

[8] W. Hochholzer and F.-J. Neumann, "Akutes Koronarsyndrom ohne ST-Streckenhebung," DMW-Deutsche Medizinische Wochenschrift, vol. 141, no. 11, pp. 782-785, 2016.

[9] J. H. Chesebro, G. Knatterud, R. Roberts et al., "Thrombolysis in myocardial infarction (TIMI) trial, phase I: a comparison between intravenous tissue plasminogen activator and intravenous streptokinase. Clinical findings through hospital discharge," Circulation, vol. 76, no. 1, pp. 142-154, 1987.

[10] G. N. Levine, E. R. Bates, J. A. Bittl et al., "2016 ACC/AHA guideline focused update on duration of dual antiplatelet therapy in patients with coronary artery disease," Journal of the American College of Cardiology, vol. 68, no. 10, pp. 1082-1115, 2016.

[11] M. Valgimigli, H. Bueno, R. A Byrne et al., "2017 ESC focused update on dual antiplatelet therapy in coronary artery disease developed in collaboration with EACTS: the Task Force for dual antiplatelet therapy in coronary artery disease of the European Society of Cardiology (ESC) and of the European Association for Cardio-Thoracic Surgery (EACTS)," European Heart Journal, vol. 39, no. 3, pp. 213-260, 2018.

[12] A. Wester, R. Attar, M. A. Mohammad et al., "Impact of baseline anemia in patients with acute coronary syndromes undergoing percutaneous coronary intervention: a prespecified analysis from the VALIDATE-SWEDEHEART trial," Journal of the American Heart Association, vol. 8, no. 16, Article ID e012741, 2019.

[13] P. V. Ennezat, S. Maréchaux, C. Pinçon et al., "Anaemia to predict outcome in patients with acute coronary syndromes,"
Archives of Cardiovascular Diseases, vol. 106, no. 6-7, pp. 357-365, 2013.

[14] P. R. Lawler, K. B. Filion, T. Dourian, R. Atallah, M. Garfinkle, and M. J. Eisenberg, "Anemia and mortality in acute coronary syndromes: a systematic review and meta-analysis," American Heart Journal, vol. 165, no. 2, pp. 143-153.e145, 2013.

[15] P. S. Levy, R. L. Quigley, and S. A. Gould, "Acute dilutional anemia and critical left anterior descending coronary artery stenosis impairs end organ oxygen delivery," The Journal of Trauma: Injury, Infection, and Critical Care, vol. 41, no. 3, pp. 416-423, 1996.

[16] S. A. McLellan and T. S. Walsh, "Oxygen delivery and haemoglobin," Continuing Education in Anaesthesia Critical Care \& Pain, vol. 4, no. 4, pp. 123-126, 2004.

[17] R. B. Weiskopf, M. K. Viele, J. Feiner et al., "Human cardiovascular and metabolic response to acute, severe isovolemic anemia," JAMA, vol. 279, no. 3, pp. 217-221, 1998.

[18] C. B. Arant, T. R. Wessel, M. B. Olson et al., "Hemoglobin level is an independent predictor for adverse cardiovascular outcomes in women undergoing evaluation for chest pain," Journal of the American College of Cardiology, vol. 43, no. 11, pp. 2009-2014, 2004.

[19] N. A. Zakai, B. French, A. M. Arnold et al., "Hemoglobin decline, function, and mortality in the elderly: the cardiovascular health study," American Journal of Hematology, vol. 88, no. 1, pp. 5-9, 2013.

[20] A. Shander, I. Gross, S. Hill, M. Javidroozi, and S. Sledge, "A new perspective on best transfusion practices," Blood transfusion, vol. 11, no. 2, pp. 193-202, 2013. 